\title{
Novel Lung Targeting Cell Penetrating Peptides as Vectors for Delivery of Therapeutics
}

\author{
Maliha Zahid ( $\nabla$ maz7@pitt.edu ) \\ University of Pittsburgh School of Medicine \\ Kayla McCandless \\ University of Pittsburgh
}

\section{Sanjay Mishra}

University of Pittsburgh

Jeffrey Stiltner

University of Pittsburgh

Kyle Feldman

University of Pittsburgh

\section{Hisato Yagi}

University of Pittsburgh School of Medicine https://orcid.org/0000-0003-1460-4043

\section{Raymond Yurko}

University of Pittsburgh

\section{Kazi Islam}

University of Pittsburgh

Jonathan Brown

MPEG LA, L.L.C.

\section{Raymond Frizzell}

University of Pittsburgh

\section{Article}

Keywords: cell penetrating peptides, lung targeting peptides, S7A, R11A

Posted Date: November 15th, 2021

DOI: https://doi.org/10.21203/rs.3.rs-1056707/v1

License: (9) This work is licensed under a Creative Commons Attribution 4.0 International License. Read Full License 


\section{Novel Lung Targeting Cell Penetrating Peptides as Vectors for Delivery of Therapeutics}

Kayla McCandless $^{1 *}$, BS, Sanjay Mishra ${ }^{2 *}$, PhD, Jeffrey Stiltner ${ }^{1}$, BS, Kyle S. Feldman ${ }^{1}$, BS, Hisato Yagi ${ }^{1}$, PhD, Raymond Yurko ${ }^{3}$, MS, Kazi Islam ${ }^{3}$, MS, Jonathan M. Brown ${ }^{4}, \mathrm{PhD}$, Raymond Frizzell $^{2}$, PhD, Maliha Zahid ${ }^{1,5}, \mathrm{MD}, \mathrm{PhD}$

1. Dept. of Developmental Biology, University of Pittsburgh School of Medicine, Pittsburgh, PA

2. Dept. of Pediatrics, University of Pittsburgh, School of Medicine, Pittsburgh, PA

3. Peptide and Peptoid Synthesis Facility, University of Pittsburgh, PA

4. Consultant, to MPEG LA, L.L.C, MD

5. Clinical and Translational Science Institute, University of Pittsburgh, PA

\section{Address for correspondence:}

Maliha Zahid, MD, Ph.D.

Department of Developmental Biology

University of Pittsburgh School of Medicine

$53045^{\text {th }}$ St., Rangos Research Center

Pittsburgh, PA15201

Phone: 412-692-8893

Fax: 412-692-6184

Email: maz7@pitt.edu

* Contributed equally to the manuscript 


\begin{abstract}
Cell penetrating peptides are unique, 5-30 amino acid long peptides that are able to breach cell membrane barriers and carry cargoes intracellularly in a functional form. Our prior work identified a synthetic, non-naturally occurring 12-amino acid long peptide that we termed cardiac targeting peptide (CTP: APWHLSSQYSRT) due to its ability to transduce cardiomyocytes in vivo. Studies looking into its mechanism of transduction identified two lung targeting peptides (LTPs), S7A (APWHLSAQYSRT) and R11A (APWHLSSQYSAT). These peptides robustly transduced human bronchial epithelial cell lines in vitro and mouse lung tissue in vivo. This uptake occurred independently of clathrin mediated endocytosis. Biodistribution studies of R11A showed peak uptake at 15 minutes with uptake in liver but not kidneys, indicating primarily a hepatobiliary mode of excretion. Cyclic version of both peptides was $\sim 100$-fold more efficient in permeating cells than their linear counterparts. As proof of principle, we conjugated anti-spike and anti-envelope SARS-CoV-2 siRNAs to cyclized R11A and demonstrate anti-viral efficacy in vitro. Our work presented here identifies two novel lung-specific cell penetrating peptides that could potentially deliver myriad therapeutic cargoes to lung tissue.
\end{abstract}




\section{Introduction}

Cell penetrating peptides (CPPs), also known as protein transduction domains, are 5-30 amino acid long peptides, capable of breaching cell membrane barriers while carrying cargoes much larger than themselves in an intact, functional form ${ }^{1,2}$. The first CPP, identified more than thirty years ago, was from the HIV coat protein, trans-activator of transcription (HIV-1 Tat), shown, in two simultaneously published papers, as having the ability to cross cell membrane barriers without a need for transfection reagents ${ }^{3,4}$. On the footsteps of these findings came the report of the third helix of Antennapedia domain as having transduction capabilities ${ }^{5}$. These abilities were mapped out to short 11-13 amino acid, cationic, arginine- and lysine-rich domains of the proteins. Not only were these peptides able to internalize full-length proteins and virus particles (e.g. HIV viral particle in the case of Tat), but they could be fused to the marker protein betagalactosidase, and internalize not only into cells, but also cross the blood-brain barrier to transduce neuronal tissue ${ }^{6}$. However, this widespread, robust and generalized transduction of all tissue types after an intra-peritoneal injection ${ }^{6}$, was an obstacle to the development of these nontissue specific CPPs as vectors for clinical use. This drawback was circumvented by the use of phage display, a Nobel prize in chemistry winning technique, developed by $\operatorname{Smith}^{7}$. Using this technique $^{8}$, investigators have identified peptides targeting tumors ${ }^{9}$, pancreatic islet cells ${ }^{10,11}$, adipose tissue $^{12}$, synovial tissue ${ }^{13}$ and cardiomyocytes ${ }^{14}$ to name a few examples.

Our prior work utilizing an in vitro and in vivo phage display methodology ${ }^{8}$ led to the identification of a unique, non-naturally occurring, 12-amino acid long cardiac targeting peptide $(\mathrm{CTP})^{14}$ that was able to robustly transduce cardiomyocytes in as little as 15 minutes after a peripheral, intra-venous injection ${ }^{15}$. This transduction ability was cardiomyocyte-specific and not species-limited, as demonstrated by us ${ }^{15}$, and others ${ }^{16,17}$. Studies into the mechanism of transduction of this peptide serendipitously identified two robust, lung targeting peptides (LTPs). In the current publication, we will detail the work leading to identification of these LTPs, biodistribution after intravenous administration, compare linear versus cyclized versions, and provide an application as vectors for delivery of siRNA targeting SARS-CoV-2 spike S and envelope E proteins. 


\section{Results}

As part of a larger set of studies investigating the mechanism of transduction of CTP (APWHLSSQYSRT), we performed an alanine scan. Briefly peptides were synthesized using solid-phase peptide synthesis with each amino acid replaced sequentially with an alanine resulting in 11 variant peptides. The original 12-amino acid CTP was synthesized as a reference along with the $6 \mathrm{~N}$-terminus CTP-A (APWHLS) and 6 C-terminus CTP-B (SQYSRT). All peptides were fluorescently labeled at the N-terminus with Cyanine 5.5 (Cy5.5), and amidecapped at the $\mathrm{C}$-terminus. Rat cardiomyoblast $\mathrm{H} 9 \mathrm{C} 2$ cells were incubated with $10 \mu \mathrm{M}$ of the peptides for 30 minutes at $37^{\circ} \mathrm{C}$, washed extensively with $\mathrm{PBS}$, trypsinized, fixed with formalin and fluorescence activated cell sorting (FACs) performed (Figure 1).

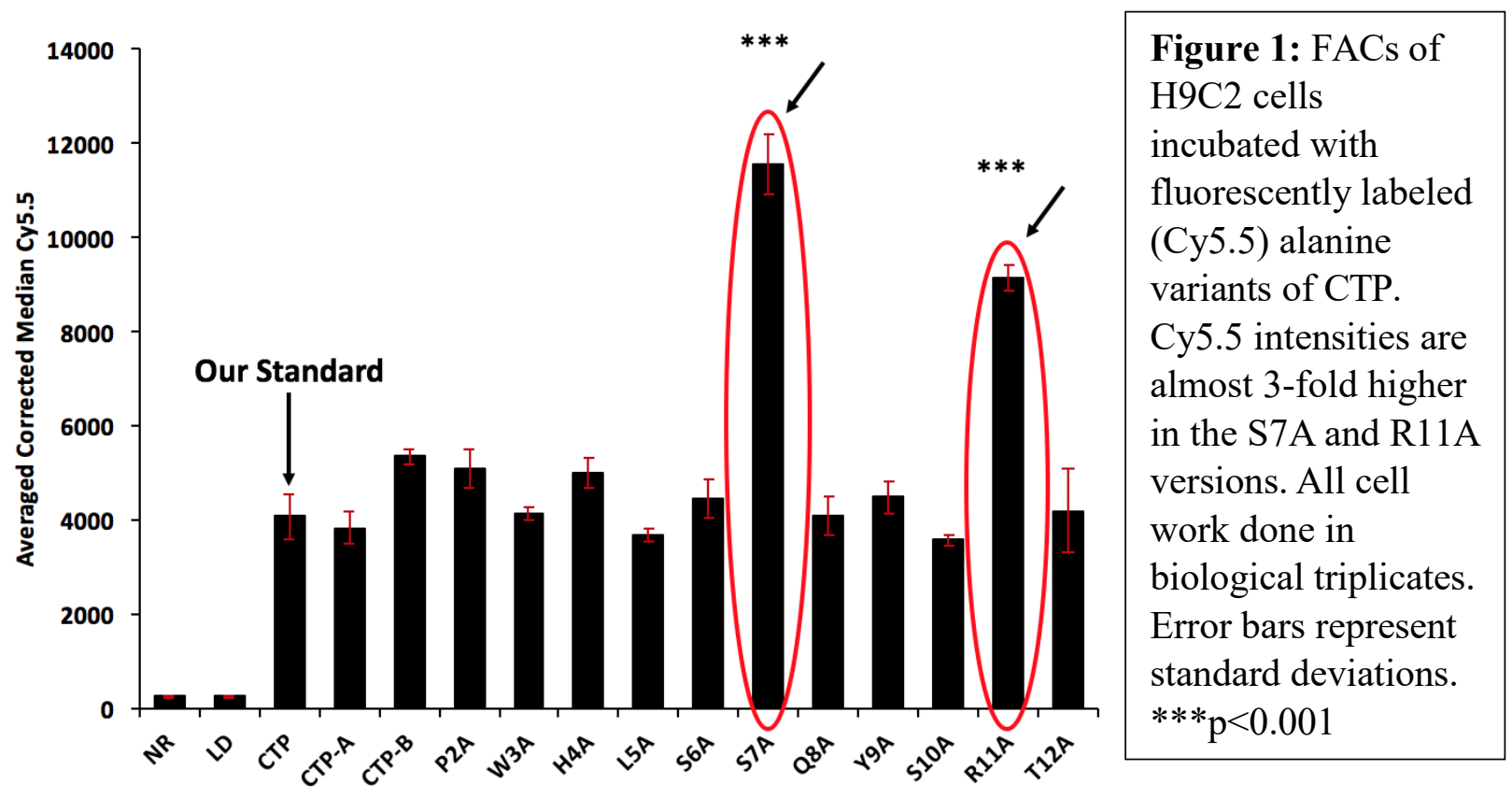

The two peptides that most robustly transduced H9C2 cells, S7A (APWHLSAQYSRT) and R11A (APWHLSSQYSAT), were administered intravenously to wild-type CD1 mice (Charles Rivers Labs) at a dose of $10 \mathrm{mg} / \mathrm{Kg}$. After 30 minutes of circulation time, the mice were euthanized using inhalational $\mathrm{CO} 2$, chests opened, and organs perfusion fixed by placing a small nick in the right atrium followed by injecting 3-5ml of $4 \%$ formalin into the left ventricular apex. Multiple organs (heart, lung, liver, kidney, spleen, brain) were harvested, fixed, cryo-sectioned, mounted, cross-stained with DAPI, and confocal imaging performed. To our surprise, the uptake in the heart was negligible, counter to our expectations based on the results of the alanine scan. Instead there was robust lung uptake of both peptides with little uptake into the liver or kidneys, and none in the heart (Figure 2: S7A top row; R11A middle row). In fact, lung uptake of R11A appeared to be even more robust than that of S7A. Decreasing doses of injected R11A still showed robust lung uptake even at the lowest dose of $1 \mathrm{mg} / \mathrm{Kg}$ (Figure 2-bottom row; Supplemental Figure 1), with improving lung to liver ratios at lower doses of R11A (Supplemental Figure 1). 


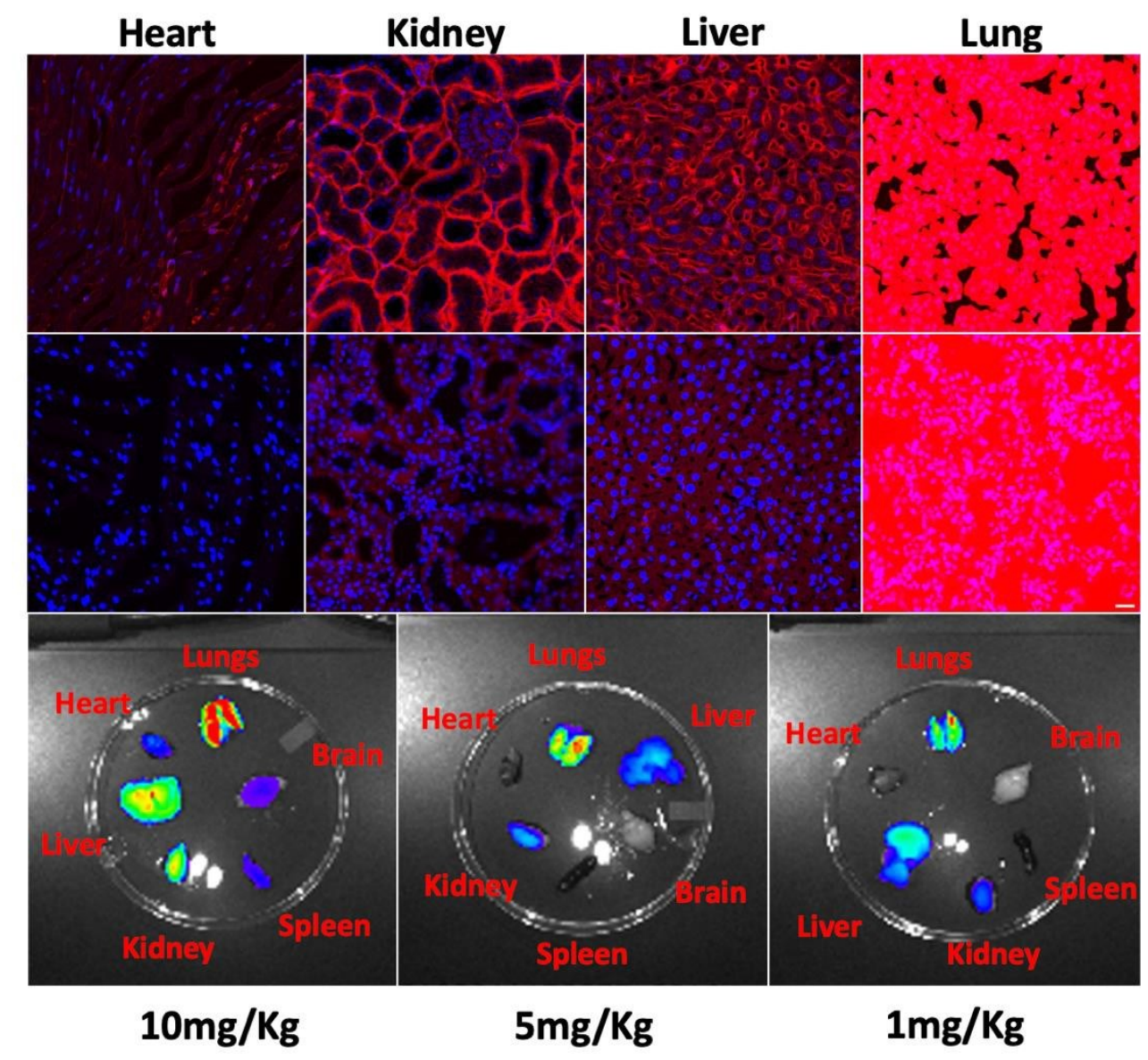

Figure 2: Uptake of fluorescently (Cy5.5) labeled S7A (top row) and R11A (middle row), $10 \mathrm{mg} / \mathrm{Kg}$, by heart, kidney, liver, and lung tissue in wild-type mice injected intravenously at 15 mins. First three panels imaged using same parameters-lungs imaged with significantly shorter exposure times to avoid saturation. Bottom Panel-Mice injected with decreasing doses of R11A, euthanized at 15 mins and ex vivo imaging of multiple organs performed using IVIS imaging system. There was robust lung uptake of R11A even at the lowest $1 \mathrm{mg} / \mathrm{Kg}$ dose. N=3. Red-Peptides; Blue-Dapi. Scale bar represents $20 \mu \mathrm{m}$.

In order to confirm uptake of these peptides by lung epithelial cells, a human bronchial epithelial cell line (CFBE410-) was grown on coverslips, incubated with $10 \mu \mathrm{M}$ of S7A, R11A or a random, scrambled peptide (RAN) for 2, 10 and 30 minutes, washed with PBS, fixed, counterstained with DAPI and confocal microscopy performed. The two LTPs (R11A and S7A) were taken up at 10 minutes with uptake increasing at 30 minutes (Supplementary Figure 2). We next undertook a biodistribution study of R11A. Wild-type, 6-week old, CD1 mice were injected with $5 \mathrm{mg} / \mathrm{Kg}$ of Cy5.5 labeled R11A peptide and allowed to circulate for 15, 30, 60, 120, 240, and 360 minutes. Mice were euthanized at the end of the circulation period, perfused and fixed as before, and multiple organs harvested and ex-vivo IVIS imaging performed immediately. Uptake by lungs was detectable and peaked at 15 minutes, the earliest time point tested. Fluorescence in liver decreased over time, with little fluorescence seen in kidneys, indicating 


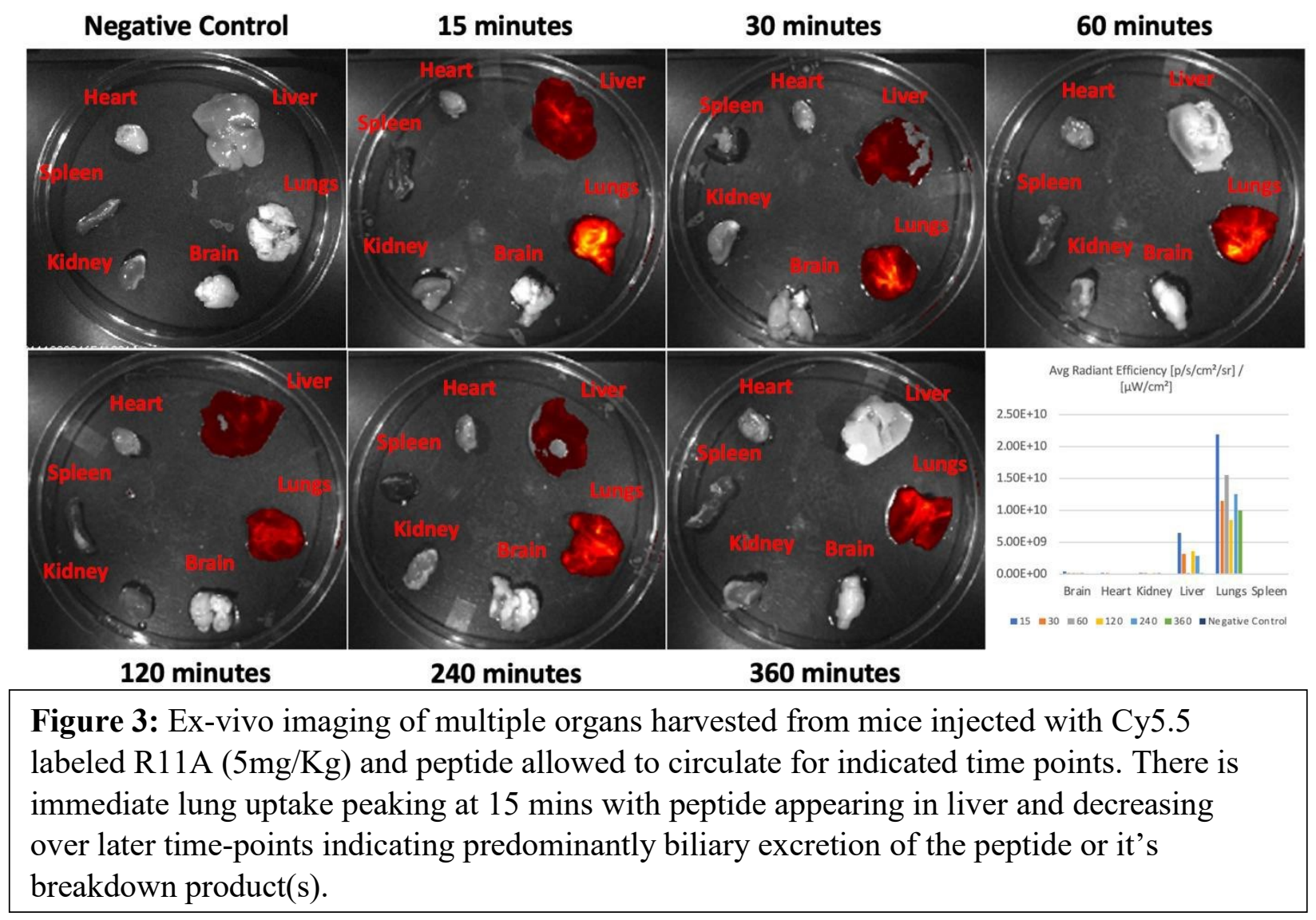

primarily a hepatobiliary mode of excretion of the peptide or its breakdown products (Figure 3; Supplemental Figure 3, n=3).

In order to investigate if the mechanism of uptake involved endocytosis, human bronchial epithelial cells were grown on coverslips, serum starved for 1 hour at $37^{\circ} \mathrm{C}$ to stimulate endocytosis, washed with cold PBS before being transferred to $4^{\circ} \mathrm{C}$ and incubated with 488 labeled transferrin along with Cy5.5 labeled S7A, R11A or a RAN peptide for one hour. After this time period, cells were either fixed, counterstained with DAPI or transferred to $37^{\circ} \mathrm{C}$ for 5 or 30 minutes before being fixed, counterstained with DAPI and confocal microscopy performed. Our results show that the peptides were internalized and did not show co-localization with the green fluorescence of transferrin that had been endocytosed. These results indicate that the peptides are being taken up by the bronchial epithelial cells in a non-clathrin dependent endocytic process (Figure 4). 


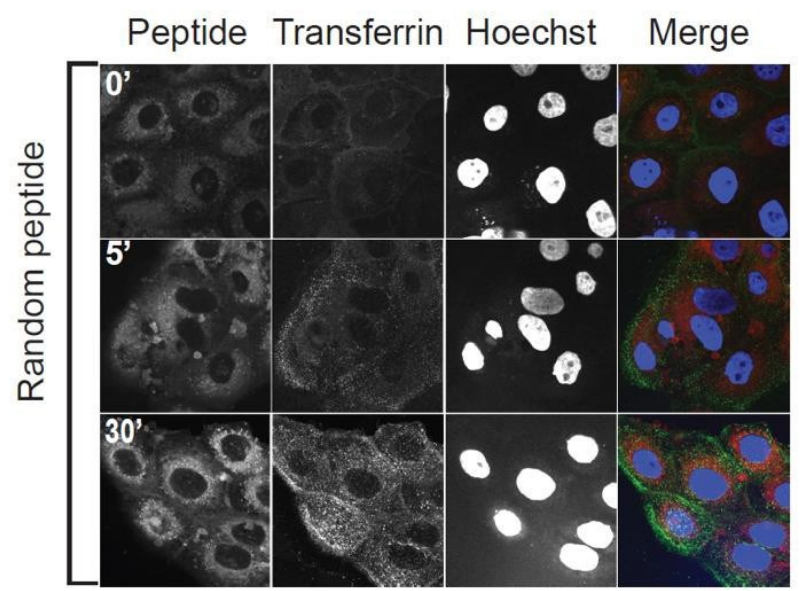

LTP Trafficking in CFBE cells

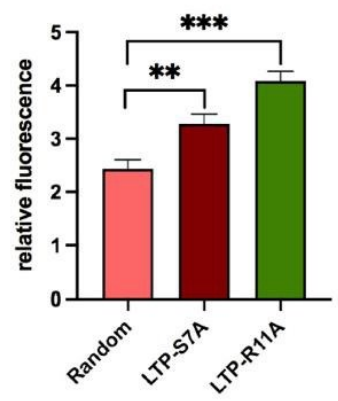

Peptide Transferrin Hoechst Merge

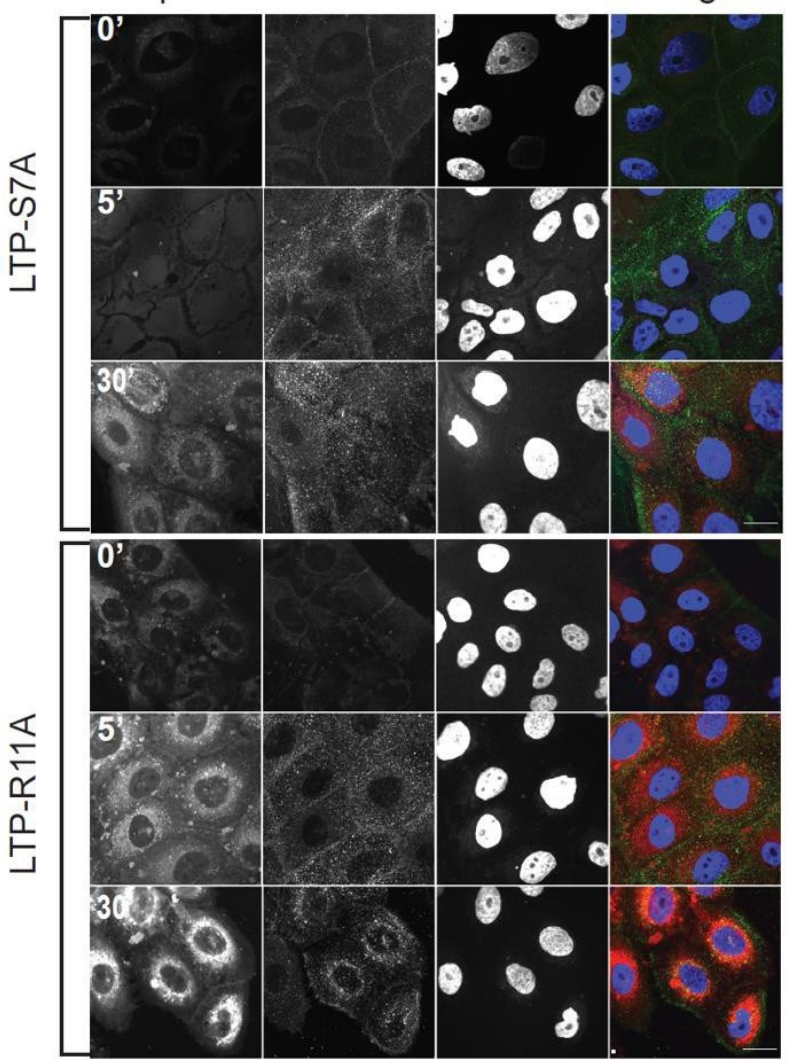

Figure 4: Bronchial epithelial cells take up LTPs in an energy independent, non-endocytic manner. Bronchial cells were serum-starved, followed by incubation with transferrin-488, or Cy5.5 labeled RAN, S7A, or R11A, followed by fixation at indicated time points, and confocal microscopy performed. Cells show very little uptake of the R11A and S7A peptide at $4{ }^{\circ} \mathrm{C}$, but increasing uptake at 5 and 30 minutes, without any co-localization with the green signal of transferrin that was endocytosed into cells. Red-Peptides; GreenTransferrin; Blue-Dapi. Scale bars represent $20 \mu \mathrm{m}$.

There have been multiple reports of cyclic versions of cell penetrating peptides having higher transduction abilities in vivo compared with their linear counterparts, likely due to better serum stability profiles ${ }^{18-20}$. In order to test whether this hypothesis applies to the present peptides, we synthesized cyclic versions of R11A and S7A by adding a lysine residue to the N-terminus, and performing a "head to tail" cyclization reaction of the terminal amino and carboxyl groups, prior to labeling the epsilon amine-group of lysine with Cy5.5. Human bronchial epithelial cell lines were incubated with linear $(1 \mu \mathrm{M})$ or cyclic peptides $(100 \mathrm{nM})$ for $30 \mathrm{mins}$, along with a yellow live-dead stain, washed extensively with pre-warmed PBS, trypsinized and FACs performed. At 10 -fold lower dose the cyclic peptides still showed a 10-fold higher cellular uptake, indicating a $\sim 100$-fold increase in transduction efficiencies (Figure 5). 

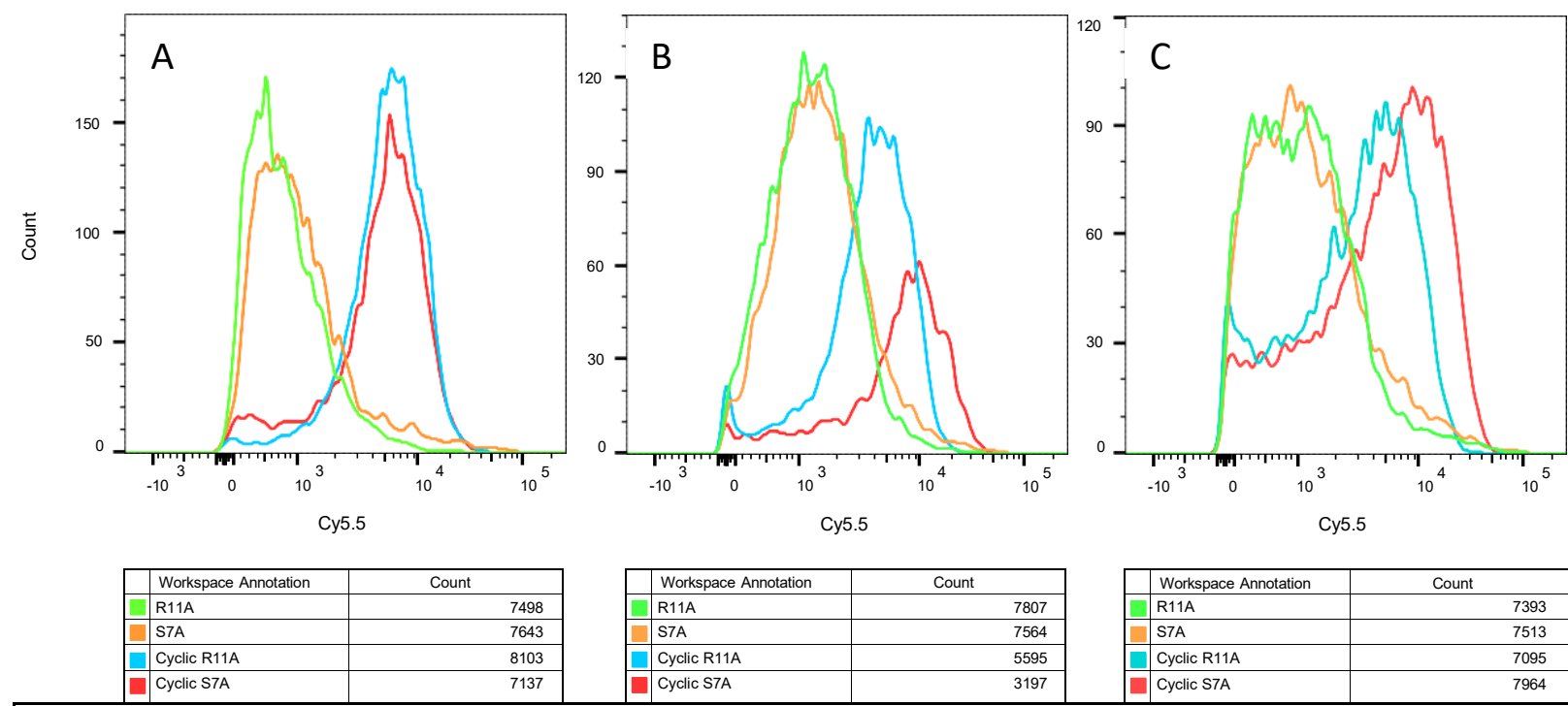

Figure 5: Cyclization of S7A and R11A increases transduction efficiencies by $~ 100$-fold. Human bronchial cells were incubated with linear $(1 \mu \mathrm{M})$ or cyclic peptides $(100 \mathrm{nM})$ and yellow fluorescent live-dead stain, and intensity of Cy5.5 fluorescence evaluated on live cells (yellow-fluorescence negative). Cyclic peptides had a roughly 10-fold increased fluorescence as compared to the linear counterparts even though the latter were incubated at a 10-fold higher concentrations. Results shown are for three separate experiments with 10,000 cells/group evaluated for each experiment.

Next, as proof of concept, our aim was to utilize cyclic R11A (cR11A) to deliver siRNA targeting various structural proteins of the SARS-CoV-2 virus as possible anti-COVID therapy. In order to design SARS-CoV-2 specific siRNA targeting key viral structural proteins, spike $\mathrm{S}$, envelope $\mathrm{E}$ and nuclear $\mathrm{N}$ proteins, we obtained SARS-CoV-2 complete genome sequence from GenBank (Accession Number: MN908947.3) ${ }^{21}$. We targeted gene-specific siRNA against SARS-CoV-2 structural proteins, and minimized off-target effects by utilizing multiple functional siRNA selection algorithms ${ }^{22-25}$ using siDirect $2.0^{26}$. Two optimized siRNAs per target protein were selected based on thermodynamic stability ${ }^{22}$ (Supplemental Table 1). These siRNAs were synthesized with a terminal thiol group and then conjugated to the side amine group of cR11A via a siRNA mono-dithio-bis-maleimidoethane (DTME) intermediate (see methods for detailed protocol). DTME contains an internal disulfide bond and has been used to link multiple siRNAs together ${ }^{27}$. Our rationale was that $\mathrm{cR} 11 \mathrm{~A}$ would internalize the siRNAs, and the disulfide bond would subsequently be cleaved in the reducing intracellular environment thereby releasing the siRNA to knock down target viral mRNA of interest via the RISC complex, and arrest viral replication. The success of the conjugation was confirmed by MALDI-Tof analysis (Supplemental Figure 4). Internalization was confirmed in human bronchial epithelial cell lines before testing the top 5 (for transduction efficiencies) in VERO cell lines that were pretreated with the cR11A-siRNA for 24 hours prior to infection with SARS-CoV-2 virus. Of the conjugates tested, cR11A-S1 and cR11A-E2, appeared to be most promising (Supplemental Table 2). Therefore, the antiviral activity of these two conjugates was evaluated further against SARS-CoV-2 (strain USA_WA1/2020) in a highly differentiated, three-dimensional, in vitro 
model of normal human bronchial cells (MatTek Corporation). These cells have unique properties in forming layers, the apical side of which is exposed only to air and has a mucin layer. Each cR11A-siRNA was tested for antiviral activity at 3 concentrations $(1.8 ; 0.18$; and $0.018 \mu \mathrm{M})$ in triplicate. cR11A-siRNA conjugates were applied to the apical and basal surface of cells for 24 hours prior to applying SARS-CoV-2 virus to the apical side only with incubation for 2 hours. Antiviral activity was measured by virus yield reduction assay in VERO 76 cells 5 days after infection. Remdesivir was included as a positive control and was tested at 4 concentrations $(2,0.2,0.02$, and $0.002 \mu \mathrm{M})$. As a virus only control, 3 of the cell wells were treated only with cell culture medium. Virus released into the apical compartment of the tissues was harvested and plated on VERO 76 cells for virus yield reduction titration. The virus yield results, EC 90 values are summarized in Figure 6. Reduction of SARS-CoV-2 titers by cR11A-S1 and cR11A-E2 occurring at a concentration of $1.8 \mu \mathrm{M}$ were statistically significant compared to virus controls. In human bronchial epithelial cells, reduction of SARS-CoV-2 titers by remdesivir at concentrations of $2,0.2$, and $0.02 \mu \mathrm{M}$ were statistically significant compared to virus controls (Figure 6). cR11A-S1 inhibited virus replication with an $\mathrm{EC}_{90}$ value of $0.64 \pm 0.2 \mu \mathrm{M}$, while cR11A-E2 inhibited virus replication with an $\mathrm{EC}_{90}$ value of $1.04 \pm 0.2 \mu \mathrm{M}$, and remdesivir inhibited virus replication with an $\mathrm{EC}_{90}$ value of $0.019 \mu \mathrm{M}$ (Figure 6b).

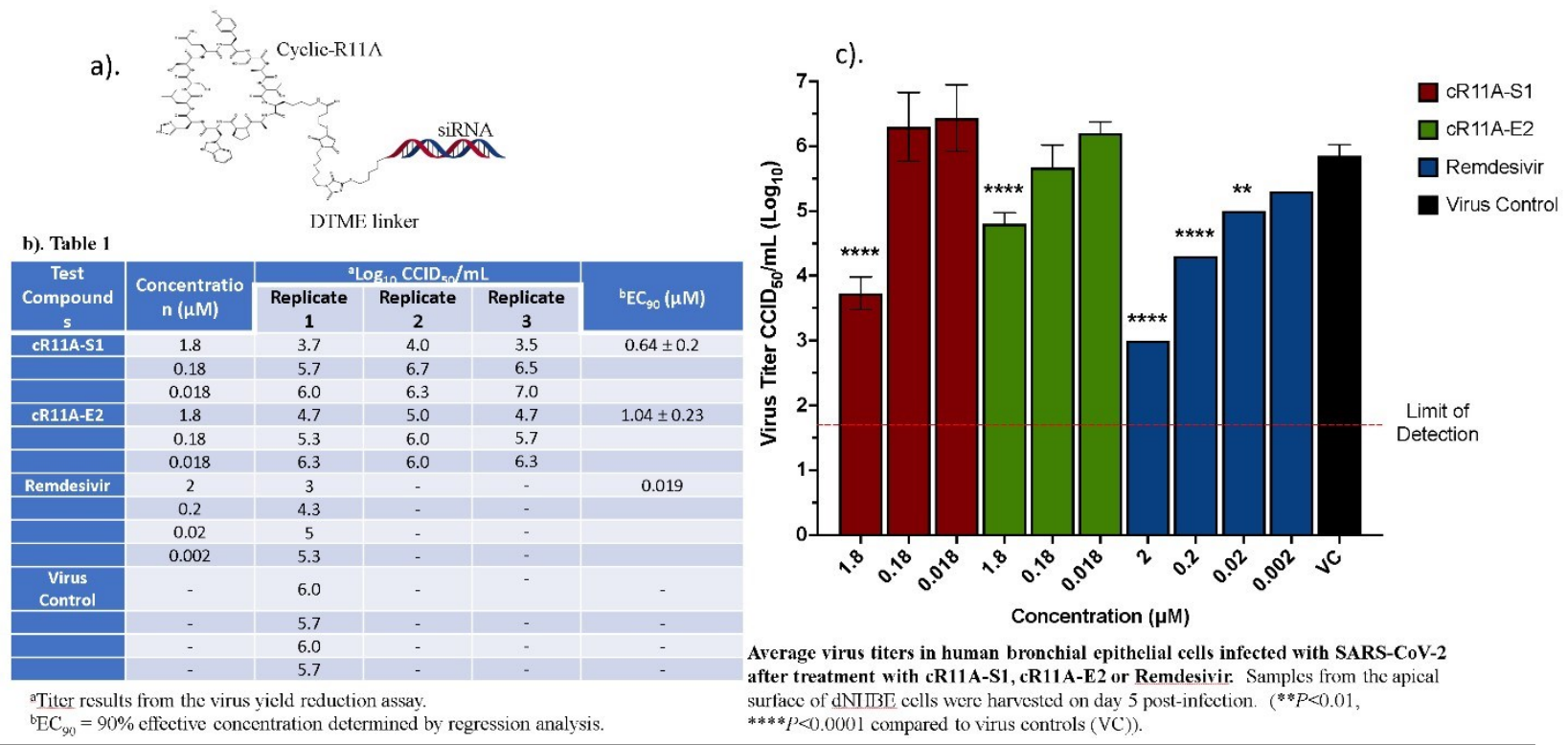

Figure 6: In vitro Antiviral Activity and EC90 Values for cR11A-siRNA-S1, cR11A-E2, and remdesivir against SARS-CoV-2 in human bronchial epithelial cell line. A). Structural representation of the $\mathrm{cR} 11 \mathrm{~A}$ along with the DTME linker linking to the siRNA represented by double-strands. B). Table showing viral titers from human bronchial epithelial cells treated with the cR11A-siRNA conjugates for 24 hours prior to infection with SARS-CoV-2 virus and the respective EC90 values, along with the EC90 value for remdesivir. C). SARS-CoV-2 viral titers plotted for $\mathrm{cR} 11 \mathrm{~A}-\mathrm{S} 1$, cR11A-E2, and remdesivir against the virus only control. All infections and assays done in triplicate. 


\section{Discussion}

In this day and age of increasing air pollution, multi-drug resistant bacteria, and evolving new viral pathogens, novel vectors targeting lung epithelium with the ability to deliver myriad cargoes would be a major breakthrough. In this current body of work, we present two synthetic, non-naturally occurring lung targeting peptides, S7A and R11A. Although this was a serendipitous finding, in retrospect, changing the amino acid sequence in the terminal half of CTP would have changed its cardiomyocyte transduction ability as indicated by the study findings of CTP-A and CTP-B, which suggest that the transduction abilities of CTP lie in its Cterminus. To the best of our knowledge these two S7A and R11A peptides are first in their class of lung epithelial targeting peptides.

A nine amino acid long cyclic peptide, CARSKNKDC, termed CAR was reported as targeting multiple layers of pulmonary arteries in a rat model of pulmonary hypertension ${ }^{28}$, and was used to deliver micelles containing fasudil, an anti-pulmonary hypertension drug, to pulmonary endothelium $^{29}$. This CPP uptake was limited to pulmonary endothelial cells, and did not target deeper lung tissue. In the pulmonary space, development of CPPs have been largely in the context of targeting lung cancers ${ }^{30-32}$. A strategy to target lung cancers takes advantage of the tumor microenvironment by targeting non-tissue specific CPPs to lung cancers by engineering CPPs that are activated in the tumor microenvironment. This was achieved by neutralizing the polycationic structure of these peptides by linking them to polyanionic peptides via a cleavable linker. These linkers were designed to take advantage of greater metalloproteinase 2 expression in the tumor microenvironment ${ }^{33}$, or greater oxidative stress leading to cleaving of the neutralizing peptide and unmasking of the $\mathrm{CPP}^{34}$.

Investigators have taken advantage of the accessibility of the lung by both systemic and inhalation routes, and have used non-cell specific penetrating peptides, like Tat peptide to deliver therapeutics of interest via the inhalational route ${ }^{35}$. Although the inhalational route has distinct advantages, like decreased dose requirement, less systemic side effects, and bypassing of the hepatobiliary system, it is not a feasible route in conditions like cystic fibrosis or primary ciliary dyskinesia. In these conditions' patients are riddled by thick mucus secretions and significant defects in mucociliary clearance, leading to mucus accumulation and drug entrapment. Another factor to consider is that a large fraction of inhaled drug are deposited in the upper airways and may not reach the terminal bronchioles or alveoli. Additionally, the uptake of drugs may be limited to the very superficial bronchiolar epithelial layer. Our two LTPs show robust uptake diffusely by lung epithelial tissue after an intravenous injection, bypassing the drawback of entrapment in mucus layers.

In order to study the mechanism of transduction, and rule out endocytosis as the mechanism of uptake, we performed serum starvation studies on human bronchial epithelial cell lines. Serum starvation was followed by co-incubation of cells with fluorescently labeled transferrin (taken up by endocytosis) vs. S7A or R11A. There was no colocalization of transferrin with the labeled Cy5.5 of the peptides, ruling out clathrin-mediated endocytosis. This was encouraging as the inside of an endocytic vesicle is still outside of the cell, and peptides with their cargoes taken up by this mechanism are likely to be degraded, and not able to exert the desired therapeutic effect. 
We chose to follow detailed biodistribution of R11A in this body of work, in preference over $\mathrm{S} 7 \mathrm{~A}$, as the former showed uptake even at the lowest $1 \mathrm{mg} / \mathrm{Kg}$ dose with lung to liver ratios increasing with lowering of the dose. Lung uptake of R11A occurred in as little as 15 mins, which is similar to CTP, where peak uptake also occurred at $15 \mathrm{mins}$, the earliest time point tested $^{15}$. We compared the cyclized version of both S7A and R11A to their linear counterparts in human bronchial epithelial cells lines in vitro, and found that cyclization increases uptake by almost 100-fold compared to the linear counterparts. Our findings are similar to that reported by others in the literature ${ }^{36-39}$, with increased transduction felt to be due to reduced conformational freedom, greater metabolic stability and higher resistance to proteolytic degradation. Due to the finding of R11A exhibiting better lung to liver ratios, and cyclic versions having significantly higher transduction efficiencies, cR11A was selected as the vector of choice to carry duplex siRNA, targeting various structural proteins of SARS-CoV-2 virus, conjugated to its N-terminus via a DTME linker. RNA interference as a therapeutic strategy against SARS-CoV-2 has been demonstrated by others ${ }^{40-43}$, utilizing lipid nanoparticles ${ }^{44,45}$, and peptide-dendrimers ${ }^{46}$. The advantage of our approach is use of a lung-specific CPP, its transduction capabilities further optimized via a cyclization strategy. Our proof-of-concept studies in human bronchial epithelial cell lines, grown in an air-liquid interface to mimic the bronchial environment, showed significant anti-viral activity for an siRNA targeting the spike protein and one targeting the envelope protein. Whether there is a synergistic effect with the use of both siRNAs, and at what doses, remains to be seen.

In this era of COVID pandemic, inhalational therapies that patients can self-administer if tested positive, or before the bronchitis/pneumonia becomes a systemic, life-threatening viremia, would be of immense value. However, in addition to testing the synergistic effects of the two identified siRNA, significant challenges will need to be overcome in the arena of aerosolized delivery of LTPs carrying siRNA. The aerodynamic diameter of the conjugates and their suspension medium would need to be $1-5 \mu \mathrm{m}^{47}$. There would be the potential for degradation of the peptide or the conjugate due to exposure to the shear force required for atomization. However, meeting these challenges could open up new forms of therapies for not just the current pandemic, but other viral pathogens like influenza A. Another advantage is the ability to swap the siRNAs for a sequence more conducive to emerging variants, since the chemistry for these $1: 1$ peptide to siRNA conjugation using a covalent, intracellularly degradable linker is now established, and hence can be applied to other CPPs and siRNAs.

In conclusion, this body of work has identified two novel lung targeting peptides that are able to deliver siRNA to human bronchial epithelial cell lines as a proof of their usefulness in delivering lung-specific therapeutics. We show that the lung is robustly transduced with lung to liver ratios improving with lower administered dose in vivo. We also show that cyclic versions of these peptides were more efficient than their linear forms. Our research has led to many questions that need to be addressed. In addition to aerosolization and potential inhalational route of delivery, the mechanism of transduction needs further study to identify potential binding partners for these unique peptides. This is not simply of academic interest as loading these peptides with cargoes could potentially mask the binding domain and interfere with cell penetration. Complete biodistribution and toxicity studies will need to be carried out for cR11A. Cyclic R11A was able 
to deliver siRNA duplexes, almost 10-fold greater than its own size, intracellularly and show a therapeutic effect in vitro. This ability to deliver cargoes many times their size in a functional form has been demonstrated before ${ }^{6}$. However, the cargo size limitation for LTPs, as there will be one, needs to be elucidated. 


\section{References}

1. Zahid M, Robbins PD. Cell-type specific penetrating peptides: therapeutic promises and challenges. Molecules 2015;20:13055-70.

2. Taylor RE, Zahid M. Cell Penetrating Peptides, Novel Vectors for Gene Therapy. Pharmaceutics 2020;12.

3. Green M, Loewenstein PM. Autonomous functional domains of chemically synthesized human immunodeficiency virus tat trans-activator protein. Cell 1988;55:1179-88.

4. Frankel AD, Pabo CO. Cellular uptake of the tat protein from human immunodeficiency virus. Cell 1988;55:1189-93.

5. Derossi D, Joliot AH, Chassaing G, Prochiantz A. The third helix of the Antennapedia homeodomain translocates through biological membranes. J Biol Chem 1994;269:10444-50.

6. Schwarze SR, Ho A, Vocero-Akbani A, Dowdy SF. In vivo protein transduction: delivery of a biologically active protein into the mouse. Science 1999;285:1569-72.

7. Smith GP. Filamentous fusion phage: novel expression vectors that display cloned antigens on the virion surface. Science 1985;228:1315-7.

8. Zahid M, Robbins PD. Identification and characterization of tissue-specific protein transduction domains using peptide phage display. Methods Mol Biol 2011;683:277-89.

9. Arap W, Pasqualini R, Ruoslahti E. Cancer treatment by targeted drug delivery to tumor vasculature in a mouse model. Science 1998;279:377-80.

10. Rehman KK, Bertera S, Bottino R, et al. Protection of islets by in situ peptide-mediated transduction of the Ikappa B kinase inhibitor Nemo-binding domain peptide. J Biol Chem 2003;278:98628.

11. Mi Z, Mai J, Lu X, Robbins PD. Characterization of a class of cationic peptides able to facilitate efficient protein transduction in vitro and in vivo. Mol Ther 2000;2:339-47.

12. Kolonin MG, Saha PK, Chan L, Pasqualini R, Arap W. Reversal of obesity by targeted ablation of adipose tissue. Nat Med 2004;10:625-32.

13. Mi Z, Lu X, Mai JC, et al. Identification of a synovial fibroblast-specific protein transduction domain for delivery of apoptotic agents to hyperplastic synovium. Mol Ther 2003;8:295-305.

14. Zahid M, Phillips BE, Albers SM, Giannoukakis N, Watkins SC, Robbins PD. Identification of a cardiac specific protein transduction domain by in vivo biopanning using a M13 phage peptide display library in mice. PLoS One 2010;5:e12252.

15. Zahid M, Feldman KS, Garcia-Borrero G, et al. Cardiac Targeting Peptide, a Novel Cardiac Vector: Studies in Bio-Distribution, Imaging Application, and Mechanism of Transduction. Biomolecules 2018;8.

16. Kim H, Mun D, Kang JY, Lee SH, Yun N, Joung B. Improved cardiac-specific delivery of RAGE siRNA within small extracellular vesicles engineered to express intense cardiac targeting peptide attenuates myocarditis. Mol Ther Nucleic Acids 2021;24:1024-32.

17. Avula UM, Yoon HK, Lee $\mathrm{CH}$, et al. Cell-selective arrhythmia ablation for photomodulation of heart rhythm. Sci Transl Med 2015;7:311ra172.

18. Nischan N, Herce HD, Natale F, et al. Covalent attachment of cyclic TAT peptides to GFP results in protein delivery into live cells with immediate bioavailability. Angew Chem Int Ed Engl 2015;54:19503.

19. Mandal D, Nasrolahi Shirazi A, Parang K. Cell-penetrating homochiral cyclic peptides as nucleartargeting molecular transporters. Angew Chem Int Ed Engl 2011;50:9633-7.

20. Cascales L, Henriques ST, Kerr MC, et al. Identification and characterization of a new family of cell-penetrating peptides: cyclic cell-penetrating peptides. J Biol Chem 2011;286:36932-43.

21. Wu F, Zhao S, Yu B, et al. A new coronavirus associated with human respiratory disease in China. Nature 2020;579:265-9. 
22. Ui-Tei K, Naito Y, Nishi K, Juni A, Saigo K. Thermodynamic stability and Watson-Crick base pairing in the seed duplex are major determinants of the efficiency of the siRNA-based off-target effect. Nucleic Acids Res 2008;36:7100-9.

23. Amarzguioui M, Prydz H. An algorithm for selection of functional siRNA sequences. Biochem Biophys Res Commun 2004;316:1050-8.

24. Reynolds A, Leake D, Boese Q, Scaringe S, Marshall WS, Khvorova A. Rational siRNA design for RNA interference. Nat Biotechnol 2004;22:326-30.

25. Ui-Tei K, Naito Y, Takahashi F, et al. Guidelines for the selection of highly effective siRNA sequences for mammalian and chick RNA interference. Nucleic Acids Res 2004;32:936-48.

26. Naito Y, Yoshimura J, Morishita S, Ui-Tei K. siDirect 2.0: updated software for designing functional siRNA with reduced seed-dependent off-target effect. BMC Bioinformatics 2009;10:392.

27. Brown JM, Dahlman JE, Neuman KK, et al. Ligand Conjugated Multimeric siRNAs Enable Enhanced Uptake and Multiplexed Gene Silencing. Nucleic Acid Ther 2019;29:231-44.

28. Urakami T, Jarvinen TA, Toba M, et al. Peptide-directed highly selective targeting of pulmonary arterial hypertension. Am J Pathol 2011;178:2489-95.

29. Gupta N, Ibrahim HM, Ahsan F. Peptide-micelle hybrids containing fasudil for targeted delivery to the pulmonary arteries and arterioles to treat pulmonary arterial hypertension. J Pharm Sci 2014;103:3743-53.

30. Lim J, Jang G, Kang S, et al. Cell-permeable NM23 blocks the maintenance and progression of established pulmonary metastasis. Cancer Res 2011;71:7216-25.

31. Qiu M, Ouyang J, Wei Y, et al. Selective Cell Penetrating Peptide-Functionalized Envelope-Type Chimeric Lipopepsomes Boost Systemic RNAi Therapy for Lung Tumors. Adv Healthc Mater 2019;8:e1900500.

32. Liu D, Angelova A, Liu J, et al. Self-assembly of mitochondria-specific peptide amphiphiles amplifying lung cancer cell death through targeting the VDAC1-hexokinase-II complex. J Mater Chem B 2019;7:4706-16.

33. Zhu L, Wang T, Perche F, Taigind A, Torchilin VP. Enhanced anticancer activity of nanopreparation containing an MMP2-sensitive PEG-drug conjugate and cell-penetrating moiety. Proc Natl Acad Sci U S A 2013;110:17047-52.

34. Weinstain R, Savariar EN, Felsen CN, Tsien RY. In vivo targeting of hydrogen peroxide by activatable cell-penetrating peptides. J Am Chem Soc 2014;136:874-7.

35. McCusker CT, Wang Y, Shan J, et al. Inhibition of experimental allergic airways disease by local application of a cell-penetrating dominant-negative STAT-6 peptide. J Immunol 2007;179:2556-64.

36. Qian Z, Martyna A, Hard RL, et al. Discovery and Mechanism of Highly Efficient Cyclic CellPenetrating Peptides. Biochemistry 2016;55:2601-12.

37. Park SE, Sajid MI, Parang K, Tiwari RK. Cyclic Cell-Penetrating Peptides as Efficient Intracellular Drug Delivery Tools. Mol Pharm 2019;16:3727-43.

38. Song J, Qian Z, Sahni A, Chen K, Pei D. Cyclic Cell-Penetrating Peptides with Single Hydrophobic Groups. Chembiochem 2019;20:2085-8.

39. Dougherty PG, Sahni A, Pei D. Understanding Cell Penetration of Cyclic Peptides. Chem Rev 2019;119:10241-87.

40. Sajid MI, Moazzam M, Cho Y, et al. siRNA Therapeutics for the Therapy of COVID-19 and Other Coronaviruses. Mol Pharm 2021;18:2105-21.

41. Tolksdorf B, Nie C, Niemeyer D, et al. Inhibition of SARS-CoV-2 Replication by a Small Interfering RNA Targeting the Leader Sequence. Viruses 2021;13.

42. Niktab I, Haghparast M, Beigi MH, Megraw TL, Kiani A, Ghaedi K. Design of advanced siRNA therapeutics for the treatment of COVID-19. Meta Gene 2021;29:100910. 
43. Gallicano GI, Casey JL, Fu J, Mahapatra S. Molecular targeting of vulnerable RNA sequences in SARS CoV-2: identifying clinical feasibility. Gene Ther 2020.

44. Idris A, Davis A, Supramaniam A, et al. A SARS-CoV-2 targeted siRNA-nanoparticle therapy for COVID-19. bioRxiv 2021.

45. Saify Nabiabad H, Amini M, Demirdas S. Specific delivering of RNAi using Spike's aptamerfunctionalized lipid nanoparticles for targeting SARS-CoV-2: A strong anti-Covid drug in a clinical case study. Chem Biol Drug Des 2021.

46. Khaitov M, Nikonova A, Shilovskiy I, et al. Silencing of SARS-CoV-2 with modified siRNApeptide dendrimer formulation. Allergy 2021;76:2840-54.

47. Gomes Dos Reis L, Svolos M, Hartwig B, Windhab N, Young PM, Traini D. Inhaled gene delivery: a formulation and delivery approach. Expert Opin Drug Deliv 2017;14:319-30. 


\section{Methods}

Peptide Synthesis: Linear Cy5.5 labeled lung targeting peptides (LTPs) were synthesized on a Liberty CEM microwave synthesizer using Fluorenylmethyloxycarbonyl (FMOC) chemistry. Stepwise addition of each FMOC protected amino acid was accomplished on an amide resin as solid support using Ethyl-(2Z)-2-cyano-2-hydroxyiminoacetate/ $N, N$ Diisopropylcarbodiimide (Oxyma/DIC) activation chemistry. The N-terminal amino group of the resin bound LTP peptides were then conjugated with Cy5.5-NHS (Lumiprobe Corporation) in DIPEA/DMF. Final cleavage of Cy5.5-LTP from the resin with Trifluoroacetic acid : Triisopropylsilane : H2O (TFA:TIPS:H2O-90:25:25) was followed by precipitation in Diethyl Ether (EtO2). The resulting crude Cy5.5-LTP peptides were purified by semi-preparative C-5 RP-HPLC on a Waters Delta Prep 4000 chromatography system using standard Acetonitrile/0.1\%TFA gradient conditions.

MALDI-TOF analysis of the purified conjugates on an Applied Biosystems Voyager workstation using $\alpha$-Cyano-4-hydroxycinnamic acid (CHCA) matrix allowed for confirmation of the expected mass and identity of the final product.

Peptide Synthesis: Cyclic Cy5.5 labeled lung targeting peptides (LTPs) were synthesized as above using 2-chlorotrityl resin as solid support. Cleavage of fully-protected peptide fragments were accomplished under mildly acidic conditions followed by head to tail cyclization using 3- (diethoxyphosphoryloxy)-1,2,3-benzotriazin-4(3H)-one (DEPBT) in Dimethylformamide/Dichloromethane (DMF/DCM) for 5 days at room temperature. Final cleavage and deprotection of the cyclized LTP peptides using Trifluoroacetic acid:Triisopropylsilane:H2O (TFA:TIPS:H2O-90:25:25) was followed by precipitation in Diethyl Ether (EtO2) to isolate the crude peptide. The epsilon amino group of Lysine was then labelled with Cy5.5-NHS in 0.1M Triethylammonium bicarbonate (TEAbc)/acetonitrile at $\mathrm{pH}$

8.5. The resulting Cy5.5 labelled cyclic LTP peptide was then directly purified by preparative C- 18 RP-HPLC on a Waters Delta Prep 4000 chromatography system using standard Acetonitrile/0.1\%TFA gradient conditions followed by lyophilization. MALDI-TOF analysis of the purified conjugates on an Applied Biosystems Voyager workstation using $\alpha$-Cyano-4hydroxycinnamic acid (CHCA) matrix allowed for confirmation of the expected mass and identity of the final product.

Peptide Synthesis: Cyclic thiolated lung targeting peptides (LTPs) were synthesized as above using 2-chlorotrityl resin as solid support. Cleavage of fully-protected peptide fragments were accomplished under mildly acidic conditions followed by head to tail cyclization using 3- (diethoxyphosphoryloxy)-1,2,3-benzotriazin-4(3H)-one (DEPBT) in Dimethylformamide/Dichloromethane (DMF/DCM) for 5 days at room temperature. Final cleavage and deprotection of the cyclized LTP peptides using Trifluoroacetic acid: Triisopropylsilane:H2O (TFA:TIPS:H2O - 90:25:25) was followed by precipitation in Diethyl Ether (EtO2) to isolate the crude peptide. The epsilon amino group of Lysine was then thiolated with 2-iminothiolane (Traut's reagent) in $0.1 \mathrm{M}$ Triethylammonium bicarbonate (TEAbc)/acetonitrile at $\mathrm{pH} 8.5$. The resulting thiolated cyclic LTP peptide was then directly purified by preparative C-18 RP-HPLC on a Waters Delta Prep 4000 chromatography system using standard Acetonitrile/0.1\%TFA gradient conditions followed by lyophilization. MALDI- TOF analysis of the purified conjugates on an Applied Biosystems Voyager workstation using $\alpha$ - Cyano-4-hydroxycinnamic acid (CHCA) matrix allowed for 
confirmation of the expected mass and identity of the final product.

Design of anti-SARS-CoV-2 siRNA: To select a target gene-specific siRNA with minimized off- target effect against SARS-CoV-2 envelope E protein and nuclear N protein, siRNAs were designed by multiple functional siRNA selection algorithms using siDirect2.0. SARSCoV-2 complete genome sequence and RNA sequence for $\mathrm{E}$ and $\mathrm{N}$ protein gene were obtained from GenBank (Accession Number: MN908947.3). We have selected the optimal 2 siRNAs per target, based on thermodynamic stability.

Conjugation of siRNA to LTPs: C6 protected siRNA oligomers (IDT technologies) were reduced to their free thiol form using DL-Dithiothreitol (DTT) in 0.1M Triethylammonium bicarbonate (TEAbc) at $\mathrm{pH} 8.5$ and then reacted with dithio-bis-maleimidoethane (DTME) in $300 \mathrm{mM} \mathrm{NaOAc} /$ acetonitrile at $\mathrm{pH}$ 5.2. Purification of the siRNA-DTME intermediate by precipitation with ethanol was followed by reaction with the purified cyclic R11A peptide in $300 \mathrm{mM} \mathrm{NaOAc/acetonitrile} \mathrm{at} \mathrm{pH} 5.2$ with gentle mixing at room temperature. Analytical C-18 RP-HPLC purification of the resulting siRNA-DTME-LTP-Cy5.5 conjugates using triethylamine acetate (TEAA)/Acetonitrile gradients on a Waters Alliance chromatography system was followed by lyophilization and then re-lyophilization from nuclease free water. MALDI-TOF analysis of the purified conjugates on an Applied Biosystems Voyager workstation using 3-hydroxypicolinic acid (3-HPA) matrix in ammonium citrate allowed for confirmation of the expected mass and identity of the final siRNA-cyclicLTP conjugate.

Fluorescence Activated Cell Sorting: Human bronchial epithelial cell lines were passaged and plated into 6-well plates at a cell density of 100,000 cells/well. After being allowed to settle for at least 48 hours, media was aspirated and replaced with media containing $1 \mu \mathrm{M}$ or $100 \mathrm{nM}$ of linear or cyclic LTPs respectively. To each well, $1 \mu \mathrm{L} / \mathrm{ml}$ of yellow Live-Dead stain was added as well. Cells were allowed to incubate for 30 mins, and then washed $3 \mathrm{x}$ with prewarmed PBS, trypsinized, trypsin neutralized and cells collected by spinning down. After one wash with PBS, cells were fixed with $2 \%$ formalin at room temperature for 10 mins, formalin washed off and cells suspended in $500 \mu \mathrm{L}$ of PBS, placed into glass round bottomed tubes and FACS performed. Untreated cells, cells treated with Live-Dead stain alone, and cells treated with $1 \mu \mathrm{M}$ of a scrambled, random peptide were used as controls. All groups were tested in biological triplicates. Gating was performed after excluding dead cells in the Live-Dead channel.

Anti-viral Testing of Cyclic R11A-siRNAs: The antiviral activity of two cyclic small interfering RNAs (siRNAs) were evaluated against SARS-CoV-2 (strain USA_WA1/2020) in a highly differentiated, three-dimensional (3D), in vitro model of normal human bronchial (dNHBE) cells. Each siRNA was tested for antiviral activity at 3 concentrations $(1.8 ; 0.18$; and $0.018 \mu \mathrm{M}$ ) in triplicate 3D inserts of wells of dNHBE cells (MatTek Corporation). Antiviral activity was measured by virus yield reduction assay in Vero 76 cells 5 days after infection. Remdesivir was included as a positive control and was tested at 4 concentrations $(2,0.2,0.02$, and $0.002 \mu \mathrm{M}$ ). The cyclic siRNAs (cR11A-S1 and cR11A-E2) were previously received as a solid and dissolved in 100\% DMSO at a concentration of $20 \mathrm{mg} / \mathrm{mL}$. The compounds were further diluted to the test dilutions in the MatTek culture medium (AIR-100-MM). Differentiated normal human bronchial epithelial (dNHBE) cells were made to order by MatTek Corporation (Ashland, MA) and arrived in kits with either 12- or 24-well inserts 
each. dNHBE cells were grown on $6 \mathrm{~mm}$ mesh disks in transwell inserts. During transportation the tissues were stabilized on a sheet of agarose, which was removed upon receipt. One insert was estimated to consist of approximately $1.2 \times 10^{6}$ cells. Kits of cell inserts (EpiAirway ${ }^{\text {TM }}$ AIR-100, AIR-112) originated from a single donor, \# 9981, a 29-yearold, healthy, non-smoking, Caucasian female. The cells have unique properties in forming layers, the apical side of which is exposed only to air and that creates a mucin layer. Upon arrival, the cell transwell inserts were immediately transferred to individual wells of a 6-well plate according to manufacturer's instructions, and $1 \mathrm{~mL}$ of MatTek's proprietary culture medium (AIR-100-MM) was added to the basolateral side, whereas the apical side was exposed to a humidified $5 \% \mathrm{CO}_{2}$ environment. Cells were cultured at $37^{\circ} \mathrm{C}$ for one day before the start of the experiment. After the $24 \mathrm{~h}$ equilibration period, the mucin layer, secreted from the apical side of the cells, was removed by washing with $400 \mu \mathrm{L}$ pre- warmed $30 \mathrm{mM}$ HEPES buffered saline solution 3 times. Culture medium was replenished following the wash steps. SARS-CoV-2 strain USA-WA1/2020 was passaged twice in Vero 76 cells to create the virus stock. Virus was diluted in AIR-100-MM medium before infection, yielding a multiplicity of infection (MOI) of approximately 0.0015 cell culture infectious dose, $50 \%$ endpoint $\left(\mathrm{CCID}_{50}\right)$ per cell.

Treatment with cyclic R11A-siRNAs $(120 \mu \mathrm{L})$ was applied to the basolateral and apical side for 24 hours prior to infection. For the infection, virus (120) was applied to the apical side for a 2- hour incubation. Following the 2-hour infection, the apical medium was removed, and the basal side was replaced with fresh compound or medium. As a virus control, 3 of the cell wells were treated with cell culture medium only. As a cell control, 3 of the cell wells were treated with cell culture medium only. The cells were maintained at the air-liquid interface for 5 days after which the medium was removed and discarded from the basal side. Virus released into the apical compartment of the tissues was harvested by the addition of $400 \mu \mathrm{L}$ of culture medium that was pre-warmed at $37^{\circ} \mathrm{C}$. The contents were incubated for $30 \mathrm{~min}$, mixed well, collected, thoroughly vortexed and plated on Vero 76 cells for virus yield reduction (VYR) titration. Triplicate and singlet wells were used for virus and cell controls, respectively.

Vero 76 cells were seeded in 96 -well plates and grown overnight $\left(37^{\circ} \mathrm{C}\right)$ to $90 \%$ confluence. Samples containing virus were diluted in 10-fold increments in MEM containing 2\% fetal bovine serum and $50 \mu \mathrm{g} / \mathrm{mL}$ of gentamicin and $200 \mu \mathrm{L}$ of each dilution transferred into respective wells

of a 96-well microtiter plate. Four microwells were used for each dilution to determine 50\% viral endpoints. After 5 days of incubation, each well was scored positive for virus if any cytopathic effect (CPE) was observed as compared with the uninfected control. The virus dose that was able to infect $50 \%$ of the cell cultures (CCID 50 per $0.1 \mathrm{~mL}$ ) was calculated by the Reed-Muench method (1938) and expressed as expressed as $\log _{10}$ CCID $50 / \mathrm{ml}$. Untreated, uninfected cells were used as the cell controls.

Animal Studies: All animal protocols were approved by the University of Pittsburgh Institutional Animal Care and Use Committee (IACUC). Wild-type, 6-week old, female CD1 mice were purchased from Charles Rivers. After at least 48 hours of acclimatization after arriving in the animal colony, mice were weighed and anesthetized with an intraperitoneal injection of a 1:1 cocktail of Ketamine:Xylazine at 1-2 $\mu \mathrm{L} / \mathrm{gm}$ body weight. After adequate anesthesia was achieved, mice were injected via tail vein with Cy5.5 labeled linear R11A and allowed to circulate for various time points. At the end of circulation time, mice were euthanized using inhalational CO2 as per IACUC-approved protocol, chest cavity opened, right atrium nicked and $3-5 \mathrm{ml}$ of $4 \%$ formalin injected through the left ventricular apex to 
perfusion fix the animals. Various organs were harvested, rinsed once in PBS and placed on a petri dish to be imaged using IVIS (Perkin- Elmer - S5 instrument). All mice were imaged using identical instrument settings (stage position, exposure time, binning, F-stop etc.).

Various organs were placed in $4 \%$ Formalin at room temperature for 4 hours, transferred to $15 \%$ sucrose at $4^{\circ} \mathrm{C}$ overnight, then $30 \%$ sucrose at $4{ }^{\circ} \mathrm{C}$ overnight, before being embedded in $50 \%$ OCT medium diluted with 50\% 1x PBS, frozen using liquid nitrogen and stored, lightprotected in $-80^{\circ} \mathrm{C}$ for later cryosectioning. Organs were sectioned at 6 micron thickness, slides made, mounted with DAPI containing mounting medium and confocal microscopy performed. All time-points were tested in at least triplicate $(n=3)$.

\section{Data Availability}

All amino acid sequences of the peptides used are provided in the manuscript. All siRNA duplexes tested are provided in the supplemental material. Detailed methods on synthesis of linear and cyclic peptides, as well as conjugation of siRNA to lysine side N-terminus of cyclic peptides is given in sufficient detail to enable reader to replicate our results.

\section{Acknowledgements}

University of Pittsburgh utilized the nonclinical/pre-clinical services offered by the National Institute of Allergy and Infectious Diseases to perform the SARS-CoV-2 work. We are also deeply grateful to Dr. Brett Hurst, Utah State University, for carrying out all of the viral work in a BSL3 environment. This work was supported by an R01 grant HL153407 from the NHLBI awarded to MZ; and with COVID Pilot Project funding from the University of Pittsburgh Clinical and Translational Science Institute and the DSF Charitable Foundation awarded to RF.

\section{Author Contributions}

$\mathrm{KC}, \mathrm{SM}$, JS, KSF, performed the cell and animal experiments. HY did the in silico dentification of anti-SARS-CoV-2 siRNA. RY, KI and JMB were responsible for peptide synthesis, cyclization and conjugation of siRNA to peptides. RF was responsible for procuring funding. $\mathrm{MZ}$ procured funding, designed experiments, interpreted data, wrote and proof-read the manuscript.

Competing Interests: MZ along with University of Pittsburgh has two patents filed on the use of lung targeting peptides: Lung-specific targeting-peptide (LTP)-mediated delivery of a therapeutic agent for treating pulmonary viral infection, US patent application No. 63/012,330, filing date April 20th, 2020; and Lung-specific targeting-peptide (LTP), composition and uses thereof, US patent application No. 62/984,925, filing date March 4th, 2020. JMB is a paid consultant to MPEG LA, L.L.C., which has a patent family pending on homo-bivalent linker compounds monosubstituted with an oligonucleotide for use in the synthesis of bioconjugates (WO 2016/205410 A2). 


\section{Supplementary Files}

This is a list of supplementary files associated with this preprint. Click to download.

- SupplementalMaterial11821.pdf 